\title{
Article \\ MCR Expression Conferring Varied Fitness Costs on Host Bacteria and Affecting Bacteria Virulence
}

\author{
Wan Li ${ }^{1,+}$, Zhihai Liu ${ }^{2,3,+}$, Wenjuan Yin ${ }^{4}$, Lu Yang ${ }^{1}$, Lu Qiao ${ }^{1}$, Shikai Song ${ }^{1}$, Zhuoren Ling ${ }^{1}$, \\ Ruicheng Zheng ${ }^{1}$, Congming $\mathrm{Wu}^{1}{ }^{1}$, Yang Wang ${ }^{1}$ and Jianzhong Shen ${ }^{1, *}$ \\ 1 Department of Veterinary Pharmacology and Toxicology, College of Veterinary Medicine, \\ China Agricultural University, Yuanmingyuan West Road No. 2, Beijing 100193, China; \\ wanli20173050431@cau.edu.cn (W.L.); yanglu2016@cau.edu.cn (L.Y.); qiaoatshandong@163.com (L.Q.); \\ songshikai@cau.edu.cn (S.S.); lingzrformal@163.com (Z.L.); b20203050400@cau.edu.cn (R.Z.); \\ wucm@cau.edu.cn (C.W.); wangyang@cau.edu.cn (Y.W.) \\ 2 College of Chemistry and Pharmaceutical Sciences, Qingdao Agricultural University, Qingdao 266109, China; \\ lzh@qau.edu.cn \\ 3 Department of Microbiology and Immunology, College of Husbandry and Veterinary Medicine, \\ Henan Agricultural University, Zhengzhou 450046, China \\ 4 Key Laboratory of Pathogenesis Mechanism and Control of Inflammatory-Autoimmune Diseases of Hebei \\ Province, College of Basic Medical Science, Hebei University, Baoding 071002, China; ywj0501@hbu.edu.cn \\ * Correspondence: sjz@cau.edu.cn \\ + These authors contributed equally to this work.
}

Citation: Li, W.; Liu, Z.; Yin, W.; Yang, L.; Qiao, L.; Song, S.; Ling, Z.; Zheng, R.; Wu, C.; Wang, Y.; et al. MCR Expression Conferring Varied Fitness Costs on Host Bacteria and Affecting Bacteria Virulence. Antibiotics 2021, 10, 872. https:// doi.org/10.3390/antibiotics10070872

Academic Editor: Sylvia Valdezate

Received: 21 May 2021

Accepted: 7 July 2021

Published: 19 July 2021

Publisher's Note: MDPI stays neutral with regard to jurisdictional claims in published maps and institutional affiliations.

Copyright: (c) 2021 by the authors. Licensee MDPI, Basel, Switzerland. This article is an open access article distributed under the terms and conditions of the Creative Commons Attribution (CC BY) license (https:// creativecommons.org/licenses/by/ $4.0 /)$.

\begin{abstract}
Since the first report of the plasmid-mediated, colistin-resistant gene, $m c r-1$, nine $m c r$ genes and their subvariants have been identified. The spreading scope of $m c r-1 \sim 10$ varies greatly, suggesting that $m c r-1 \sim 10$ may have different evolutionary advantages. Depending on MCR family phylogeny, $m c r-6$ is highly similar to $m c r-1$ and -2 , and $m c r-7 \sim 10$ are highly similar to $m c r-3$ and -4 . We compared the expression effects of MCR-1 5 on bacteria of common physiological background. The MCR-1-expressing strain showed better growth than did MCR-2 5-expressing strains in the presence of colistin. LIVE/DEAD staining analysis revealed that MCR-3 5 expression exerted more severe fitness burdens on bacteria than did MCR-1 and -2. Bacteria expressing MCRs except MCR-2 showed enhanced virulence with increased epithelial penetration ability determined by trans-well model $(p<0.05)$. Enhanced virulence was also observed in the Galleria mellonella model, which may have resulted from bacterial membrane damage and different levels of lipopolysaccharide (LPS) release due to MCR expression. Collectively, MCR-1-expressing strain showed the best survival advantage of MCR-1 5-expressing strains, which may partly explain the worldwide distribution of $m c r-1$. Our results suggested that MCR expression may cause increased bacterial virulence, which is alarming, and further attention will be needed to focus on the control of infectious diseases caused by $m c r$-carrying pathogens.
\end{abstract}

Keywords: $m c r-1 \sim 5$; fitness cost; virulence; prevalence

\section{Introduction}

Colistin is considered a "last-resort" antibiotic for treating infections caused by multidrug-resistant bacteria. However, the therapeutic effect of colistin has been greatly compromised by the emergence of the mobile colistin-resistance gene mcr-1 [1]. mcr-1positive bacteria have been detected worldwide from humans, animals, the environment, and aquatic sources [2-5]. The mor gene family has expanded to different bacterial species, such as Escherichia coli in Belgium (mcr-2) [4], Enterobacteriaceae and Aeromonas in China ( mcr-3) [5], Salmonella and E. coli in Spain and Belgium (mcr-4) [6], Salmonella in Germany (mcr-5) [7], Moraxella spp. in the United Kingdom (mor-6) [8], K. pneumoniae in China (mcr-7, -8) [7,9], Salmonella enterica serotype Typhimurium in the USA (mcr-9) [10], and 
Enterobacter roggenkampii in China (mcr-10) [9]. mor genes have a large and diverse epidemiological distribution, while $m c r-1$-positive bacteria have been reported across six continents. However, the distribution of the other $m c r$ genes $(m c r-2 \sim 10)$ is relatively limited [11]. Whether the fitness costs for bacteria caused by mor genes can influence the gene's dissemination remains uncertain.

The biological costs imposed by a plasmid-mediated resistance gene affect its maintenance in the bacterial community, because resistant strains with the extra burden could easily be replaced by susceptible strains in the absence of antibiotic pressure [12,13]. Previous studies have verified that $m c r$ gene expression affected bacterial growth conditions [12] Expression of MCR-1 and MCR-3 exerted fitness costs on the first 50 generations of host bacteria, and compensatory mutations can alleviate these costs [12]. mor-carrying plasmids with different replicon types are varied in their fitness burdens on host bacteria. The IncI2, IncHI2, and IncX4 plasmid-carrying $m c r-1$ genes are stable and hardly affect bacterial growth, which explains why most reported $m c r-1$-bearing plasmids belong primarily to these types [12,13]. Although the fitness costs of $m c r-1$-carrying strains have been thoroughly studied under different conditions, few studies have compared the fitness costs of different $\mathrm{mcr}$ genes in a common physiological background.

MCR transfers the phosphoethanolamine ( $\mathrm{pEtN}$ ) on lipid A, alters its negative charge, and decreases the colistin-binding efficiency. However, MCR-mediated lipid A modification and its subsequent effect on bacterial virulence remain largely unexplored. It is reported that there is a complicated relationship between colistin resistance and bacterial virulence [14]. Increased colistin resistance along with decreased virulence was observed in E. coli [1]. However, some A. baumannii exhibited increased colistin resistance but also increased virulence gene expression [15]. Worryingly, some pathogenic clonal lineages, such as E. coli ST131, have been isolated from clinical patients, food animals, and environments harboring $m c r$, which is alarming because carrying of $m c r$ in this clonal may not only result in therapeutic failure, but also varied virulence to host animal [16-18]. All this indicates that it is necessary to figure out the association between MCR mediated colistin resistance and virulence variation in bacteria.

Herein, in this study, we analyzed the fitness costs and virulence of bacteria carrying $m c r-1 \sim 5$, which contributed to exploring the causes of $m c r$ genes' widespread distribution and was a reminder of the risk of $m c r$-carrying bacteria outbreak.

\section{Materials and Methods}

\subsection{Bacterial Strains, Plasmids, Cell Lines, and Culture Conditions}

Bacterial strains, plasmids, and cell lines used in this study are listed in Table 1. Bacteria carrying $m c r-1 \sim 5$ were isolated previously and kept in our lab. Strains were grown in LB broth (Luqiao, Beijing, China) under $37^{\circ} \mathrm{C}$ with proper antibiotics. E. coli TOP10 (mcr-1 5/pBAD) was constructed by cloning $m c r-1 \sim 5$ genes in frame into arabinose-inducible pBAD-hisA expression vector with primers listed in Table A1; hence, the expression of MCR-1 5 can be regulated with L-arabinose (Sigma-Aldrich, St. Louis, MO, USA) induction. E. coli TOP10 transformed with empty pBAD vector was used as the control group. Bacteria were cultured in Luria-Bertani (LB) medium (Luqiao, Beijing, China) at $37^{\circ} \mathrm{C}$. The growth medium was supplemented with antibiotic and/or L-arabinose.

Cell line Caco-2 was grown in 12 well trans-well cell culture inserts (Nunc, Rochester, NJ, USA) to confluency. The inserts were cultured $18-22$ days at $37{ }^{\circ} \mathrm{C}, 10 \% \mathrm{CO}_{2}$ in minimum essential medium (MEM, Gibco, Waltham, MA, USA) supplemented with fetal bovine serum (FBS, Gibco, Waltham, MA, USA), 1\% non-essential amino acids (Gibco, Waltham, MA, USA), and 1\% penicillin/streptomycin (Gibco, Waltham, MA, USA). The cell culture medium was changed every other day until the cells were fully differentiated. 
Table 1. Bacteria and cells used in this study.

\begin{tabular}{|c|c|c|}
\hline Name & Description & Source or Reference \\
\hline \multicolumn{3}{|l|}{ Strains } \\
\hline SHP45 & WT E. coli strain with $m c r-1$ of pig origin & [17] \\
\hline $\mathrm{DH} 5 \alpha-m c r-2$ & Constructed E. coli strain with $m c r-2$ & {$[18]$} \\
\hline WJ1 & WT E. coli strain with $m c r-3$ of pig origin & {$[19,20]$} \\
\hline D32 & WT E. coli strain with $m c r-4$ of pig origin & Laboratory collection \\
\hline D13 & WT E. coli strain with $m c r-5$ of pig origin & Laboratory collection \\
\hline$m c r-1 / \mathrm{pBAD}$ & E. coli TOP10 with $m c r-1 / \mathrm{pBAD}$ & This study \\
\hline$m c r-2 / \mathrm{pBAD}$ & E. coli TOP10 with $m c r-2 / \mathrm{pBAD}$ & This study \\
\hline$m c r-3 / \mathrm{pBAD}$ & E. coli TOP10 with $m c r-3 / \mathrm{pBAD}$ & This study \\
\hline$m c r-4 / \mathrm{pBAD}$ & E. coli TOP10 with $m c r-4 /$ pBAD & This study \\
\hline$m c r-5 / \mathrm{pBAD}$ & E. coli TOP10 with $m c r-5 / \mathrm{pBAD}$ & This study \\
\hline pBAD & E. coli TOP10 with pBAD & This study \\
\hline ATCC25922 & E. coli, quality control strain & Laboratory collection \\
\hline \multicolumn{3}{|l|}{ Cells } \\
\hline Caco-2 & Human colon carcinoma cell line & Laboratory collection \\
\hline
\end{tabular}

\subsection{Antimicrobial Susceptibility Testing}

Colistin susceptibility of constructed strains was assessed by broth microdilution according to the European Committee on Antimicrobial Susceptibility Testing (EUCAST). Briefly, cells were resuspended to $0.5 \mathrm{McF}$ arland standard and used to inoculate 96-well microtiter plates (Corning Inc., Corning, NY, USA) containing cation-adjusted MuellerHinton medium (Luqiao, Beijing, China) with colistin $(0.06 \sim 128 \mu \mathrm{g} / \mathrm{mL})$. L-arabinose was added to experimental wells to a final concentration of $0 \sim 0.2 \%(w / v)$. Plates were incubated $16 \sim 18 \mathrm{~h}$ at $37^{\circ} \mathrm{C}$. Experiments were carried out in duplicate.

\subsection{Bacteria Growth Kinetics Detection}

Bacteria were streaked on LB agar supplemented with ampicillin. Single clones were inoculated into $1 \mathrm{~mL}$ of LB broth supplemented with $100 \mathrm{mg} / \mathrm{L}$ ampicillin and shaken at $37^{\circ} \mathrm{C}$ for $6 \mathrm{~h}$ to $\log$ phase. The bacteria were diluted to a turbidity of 0.5 , which was diluted 100 times in LB broth with L-arabinose $0.02 \%(w / v)$. Bacteria only or bacteria as well as drugs were added to the 96-well plate then placed in a microplate reader and cultured at $37^{\circ} \mathrm{C}$. The growth state of the bacteria was monitored for $24 \mathrm{~h}$, and the OD value of each well was measured intermittently using a wavelength of $600 \mathrm{~nm}$. The growth curves of the bacteria were fitted using GraphPad prism by triplicate growth kinetics assays.

\subsection{Confocal Microscopy Imaging Using LIVE/DEAD Staining}

Strains were grown overnight in LB broth supplemented with $100 \mathrm{mg} / \mathrm{L}$ ampicillin at $37^{\circ} \mathrm{C}(120 \mathrm{rpm})$. Overnight cultures were standardized to OD600 0.05 and inoculated $(1: 10 ; v / v)$ into fresh LB broth for $2 \mathrm{~h}\left(37^{\circ} \mathrm{C} ; 120 \mathrm{rpm}\right)$. The L-arabinose $(0.02 \%, w / v)$ was added and further incubated for $2 \mathrm{~h}$. The strains were stained with $6 \%$ LIVE/DEAD ${ }^{\circledR}(v / v$; BacLightTM Bacterial Viability Kit, Invitrogen, CA, USA) in phosphate-buffered saline before CLSM imaging (Leica TCS SP8, Leica, Wetzlar, Germany) with a $\times 63$ lens. The CLSM images were analyzed using ImageJ version 1.52a analysis software.

\subsection{Membrane Permeability Test Assay}

Strains were inoculated in LB broth with $0.02 \% \mathrm{~L}$-arabinose and cultured at $37^{\circ} \mathrm{C}$, $180 \mathrm{rpm}$ for $2 \mathrm{~h}$. NPN (1-N-phenylnaphthylamine, Sigma-Aldrich, St. Louis, MO, USA) with final concentration at $10 \mu \mathrm{M}$ was added and incubated at $37{ }^{\circ} \mathrm{C}$ for $30 \mathrm{~min}$ in the dark; fluorescence was detected at the excitation and emission wavelengths of 350 and $420 \mathrm{~nm}$, respectively. 


\subsection{Cell Infection Experiment}

To detect bacterial ability to penetrate the epithelial barrier, we challenged the apical surface of Caco- 2 monolayers by the addition of $1 \times 10^{6} \mathrm{CFU} / \mathrm{mL}$ strains with or without $m c r$ genes after L-arabinose induction. After incubation at $37^{\circ} \mathrm{C}$, the lower cell liquid was gathered and inoculated on LB agar. After overnight incubation, CFU on agar was counted. Every time before and after the experiment, $m c r$ genes were detected by PCR with primers pBAD-F (5'ATGCCATAGCATTTTTATCC $\left.3^{\prime}\right)$ and pBAD-R (5'GATTTAATCTGTATCAGG3') to make sure the gene is still maintained in host bacteria.

\subsection{G. mellonella Infection Model}

In vivo virulence of strains was evaluated using G. mellonella infection model, as described by Qiue [1]. Briefly, bacterial pellets were washed and diluted to an appropriate density with PBS. We injected $10 \mu \mathrm{L}$ aliquots of serially diluted bacterial suspension $\left(10^{8} \mathrm{CFU} / \mathrm{mL}\right)$ into the hemocoel of each larvae through the rear left proleg. A group of 5 larvae was randomly chosen to inject inoculation in triplicate. After injection, larvae were incubated at $37^{\circ} \mathrm{C}$, and the melanization of larvae were observed $1 \mathrm{~h}$ after infection. In all cases, no dead larvae were observed in the control groups.

After G. mellonella was ground, the homogenate was diluted in gradient and the bacteria was removed by filtration with $0.2 \mu \mathrm{m}$ filters. The samples were quantified for the presence of LPS using the LAL. Briefly, samples and LPS standards (Sigma-Aldrich, St. Louis, MO, USA) were diluted to the concentration between 0.1 and 1 endotoxin unit and incubated for $10 \mathrm{~min}$ with LAL lysates (Thermo Scientific ${ }^{\mathrm{TM}}$, Waltham, MA, USA) at $37^{\circ} \mathrm{C}$. Then, the samples with LAL lysates were treated with chromogenic reagent and color stabilizer reagents. LPS levels were determined by detecting absorptions at $405 \mathrm{~nm}$.

\subsection{Statistical Analysis}

All assays were repeated three times. Data are expressed as the mean \pm standard error of the mean (SEM). Statistical significance $\left({ }^{* * *}, p<0.001 ;{ }^{* *}, p<0.01 ;{ }^{*}, p<0.05\right.$; ns (not significant)) was calculated via two tailed $t$-test to compare treatment/experimental and control groups. Analyses was performed by using GraphPad prism 8.0 (GraphPad Software, La Jolla, CA, USA).

\section{Results}

\subsection{Mcr-1 5 Mediated Colistin Resistance Levels}

To compare $\mathrm{mcr}$-mediated resistance to colistin in a common physiological background, we expressed MCR in TOP10 E. coli by cloning mor into pBAD expression vectors, which was confirmed by PCR and sequencing (Table 1). MCR expression was induced under different $\mathrm{L}$-arabinose concentrations after $2 \mathrm{~h}$ of inoculation in LB broth. As the concentration of L-arabinose increased within $0 \sim 0.02 \%(w / v)$, the minimum inhibitory concentrations (MICs) against colistin increased (Figure 1). However, when the L-arabinose concentration reached $0.2 \%$, the MICs for colistin of the mcr-1 3-carrying strains were the same as those at $0.02 \%$, while those of $m c r-4$ and -5 were decreased by half $(1 \mathrm{mg} / \mathrm{L})$. All constructed strains exhibited maximal MICs $(2 \mathrm{mg} / \mathrm{L})$ to colistin under $0.02 \%$ L-arabinose, which was 8 32 times higher than the MICs without L-arabinose induction, and no higher MICs were observed. Therefore, we used $0.02 \%$ L-arabinose as the induction concentration in subsequent experiments.

\subsection{MCR-1 5 Expression Conferred Varied Fitness Costs on the Host Strain}

To determine the impact of MCR-1 5 expression on bacterial growth, we constructed growth curves in the presence/absence of $1 \mathrm{mg} / \mathrm{L}$ colistin. All data were fitted with logistic regression to show the host strain growth phase. The bacterial growths of MCR-3, -4 , and -5 overexpression without colistin were significantly inhibited in the log phase $(p<0.01)$ compared with that of the control group, while MCR-1 imposed a slight fitness 
disadvantage in the lag phase $(p<0.05$; Figure 2). Surprisingly, MCR-2 showed a similar growth kinetic to that of the host strain (Figure 2a).

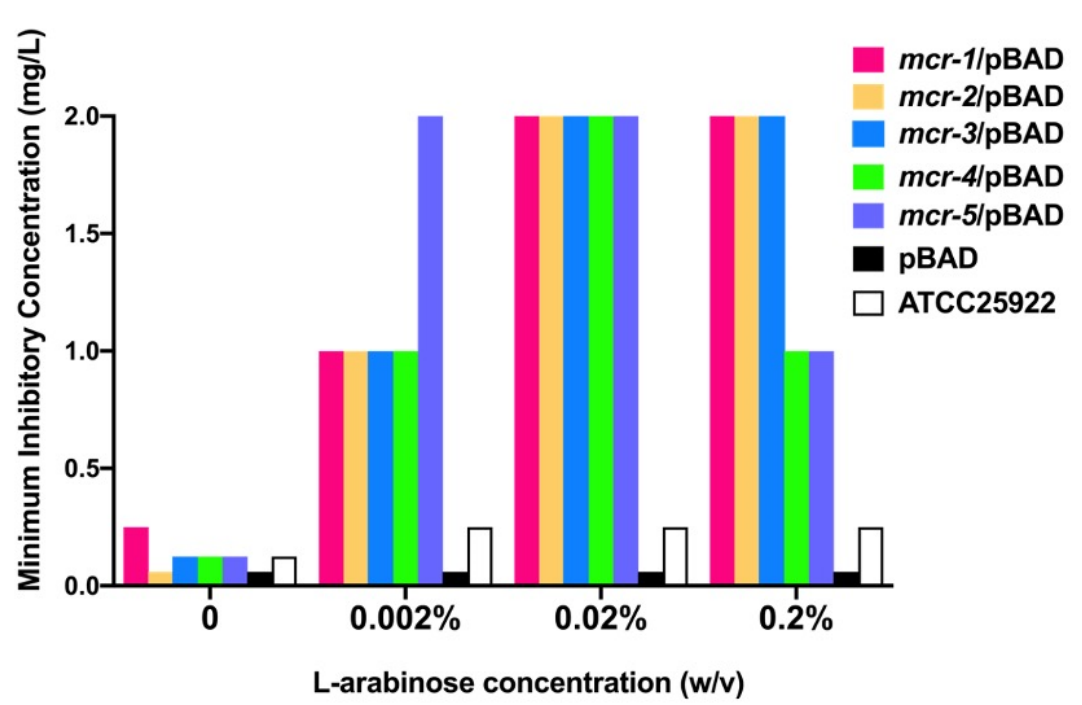

Figure 1. MICs of colistin to constructed strains in the presence of $0-0.2 \%(w / v)$ L-arabinose in broth.

a

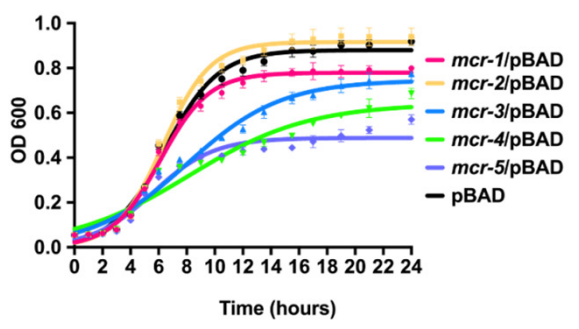

C

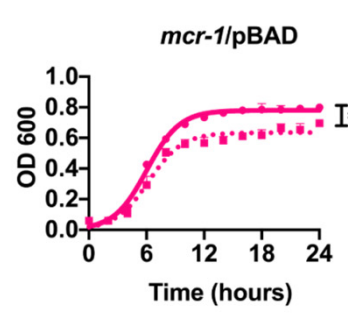

f

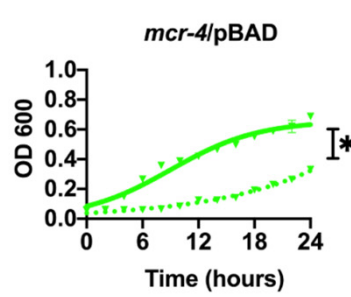

d

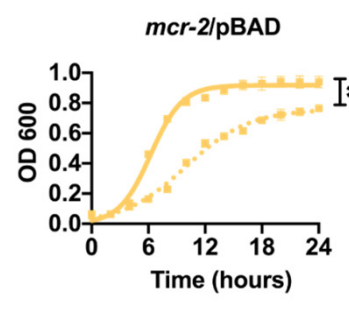

g

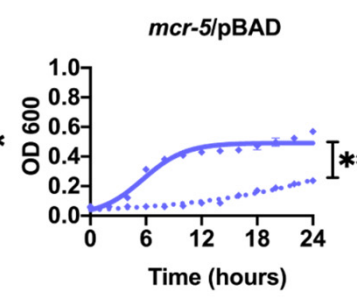

b

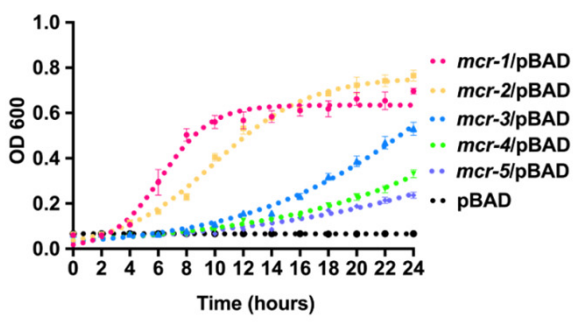

e $m c r-3 / p B A D$

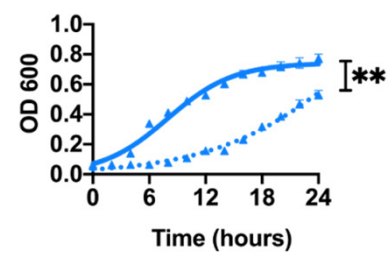

h

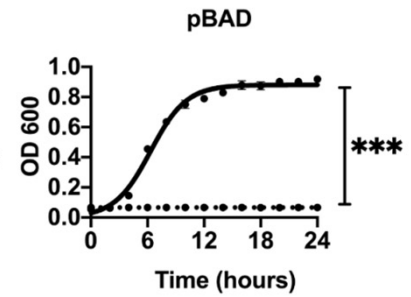

Figure 2. Growth kinetics of MCR-expressing strains in the presence or absence of $0.5 \mathrm{mg} / \mathrm{L}$ colistin in broth. (a) Growth kinetics of bacteria without colistin and (b) with $1 \mathrm{mg} / \mathrm{L}$ colistin added to the medium. (c-h) Comparisons of each MCR-expressing strain with or without colistin added to the medium. Solid lines represent the absence of colistin; dashed lines represent addition of colistin to the medium. ${ }^{*} p<0.05,{ }^{* *} p<0.01,{ }^{* * *} p<0.001$. 
MCR-1- and MCR-2-expressing strains could grow with different kinetics in the log phase with $1 \mathrm{mg} / \mathrm{L}$ colistin. However, the growths of strains expressing MCR-3, -4 , and -5 were significantly inhibited in the log phase $(p<0.01)$, and no normal growth curves could be detected after $24 \mathrm{~h}$ (Figure 2c,g). Interestingly, when colistin was added, MCR-2expressing strains showed poorer growth than did MCR-1-expressing strains (Figure 2c,d) in the logarithmic phase.

We performed LIVE/DEAD double-staining assays to reflect cell viability (Figure 3a-f). Compared with the control bacteria that only harbored PBAD, MCR-1, $-3,-4$, and -5 expressions caused significantly high ratios of dead bacteria to all bacteria $(p<0.05)$, while MCR-2 expression yielded comparable ratios to those of the control group (Figure 3g).
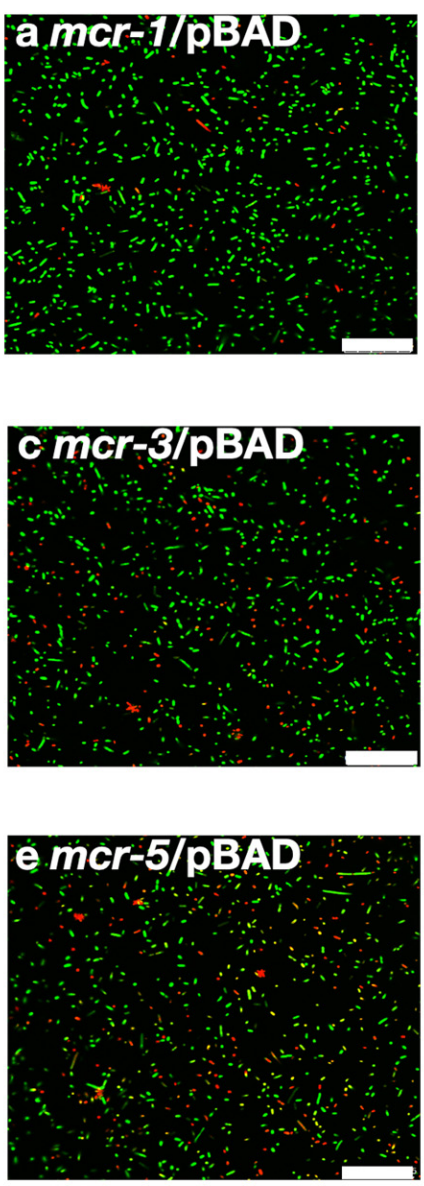
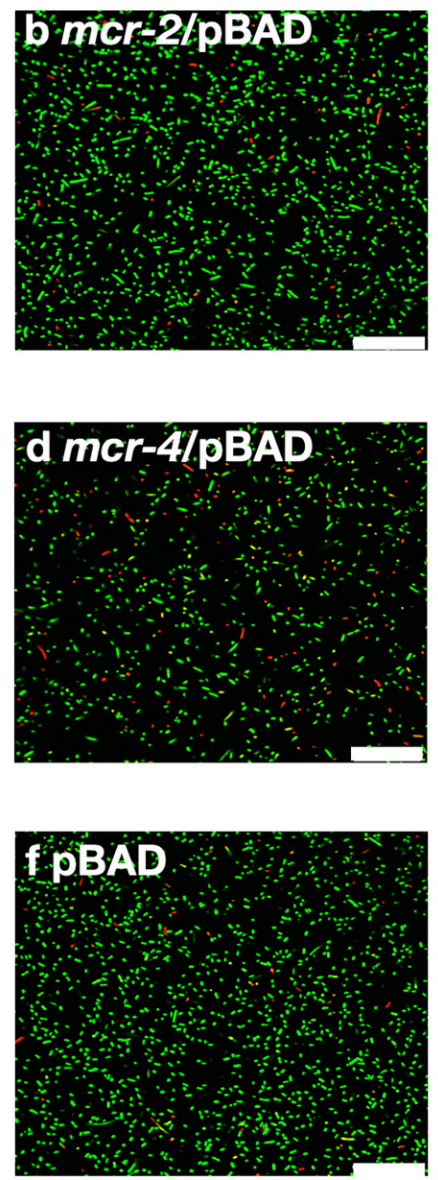

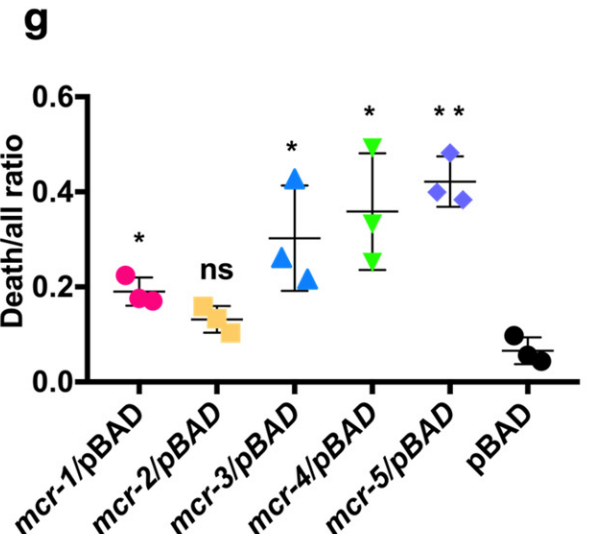

h

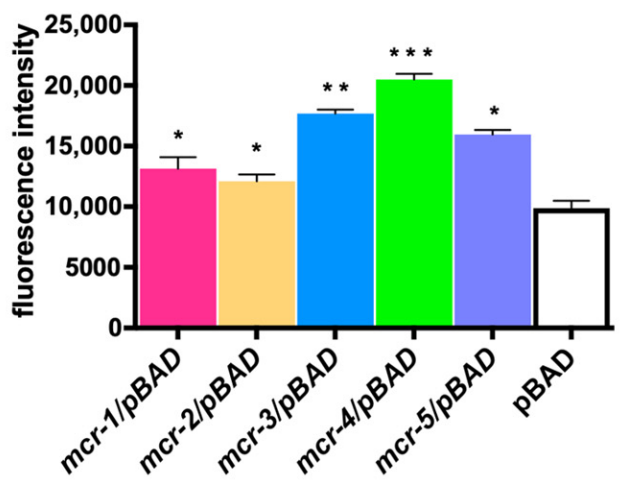

Figure 3. LIVE/DEAD staining of strains expressing MCR genes. (a-f) Confocal observation of strains expressing MCR-1-5 with $0.02 \%$ L-arabinose induction. (g) Statistical analysis of LIVE/DEAD staining images; images were analyzed using ImageJ software. (h) Membrane permeability of strains expressing MCR genes. Fluorescence intensity of NPN after 5 min of incubation with strains was detected at $420 \mathrm{~nm} .{ }^{*} p<0.05,{ }^{* *} p<0.01$, ${ }^{* * *} p<0.001$, ns: no significant difference. Scale bars represent $25 \mu \mathrm{m}$.

Because 1-N-phenylnaphthylamine (NPN) is hydrophobic, it cannot penetrate intact membranes, whereas NPN uptake may be enhanced in bacteria with damaged outer membranes. We detected the outer membrane permeability of the strains after L-arabinose induction by measuring the NPN uptake in bacteria. The strains expressing MCR-1 5 showed significantly increased membrane permeability $(p<0.05)$, with the order of membrane damage being MCR-4 > MCR-3 > MCR-5 > MCR-1 > MCR-2. Membrane permeability in the MCR-2-expressing strains was similar to that of the control bacteria (Figure $3 \mathrm{~h}$ ). 


\subsection{MCR Expression Increased Bacterial Virulence In Vitro and In Vivo}

Intestinal epithelial cells can generate various types of barriers to protect the intestinal mucosa from commensal and pathogenic microorganisms. Here, we explored whether MCR-catalyzed lipid A modification could affect the ability of bacteria to penetrate the epithelial barrier via a trans-well model in vitro (Figure 4a). The translocated bacteria were quantified by counting the viable cells. Bacteria expressing MCR-1, $-3,-4$, and -5 showed a significantly higher capacity to translocate the Caco-2 monolayer than did the control group $(p<0.05)$, where MCR-3-expressing strains showed the highest capacity to penetrate the epithelial barrier. However, MCR-2-expressing strains showed a similar penetration capacity to the non-pathogenic PBAD control group (Figure 4b). MCR-1 expressing strain showed intermediate ability to penetrate Caco-2 monolayer, which is higher than MCR-2 but lower than MCR-3 5-expressing strains.

a

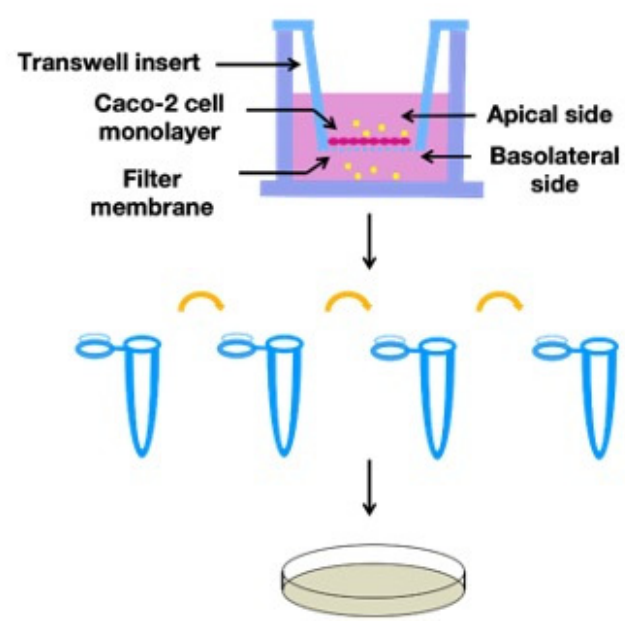

b

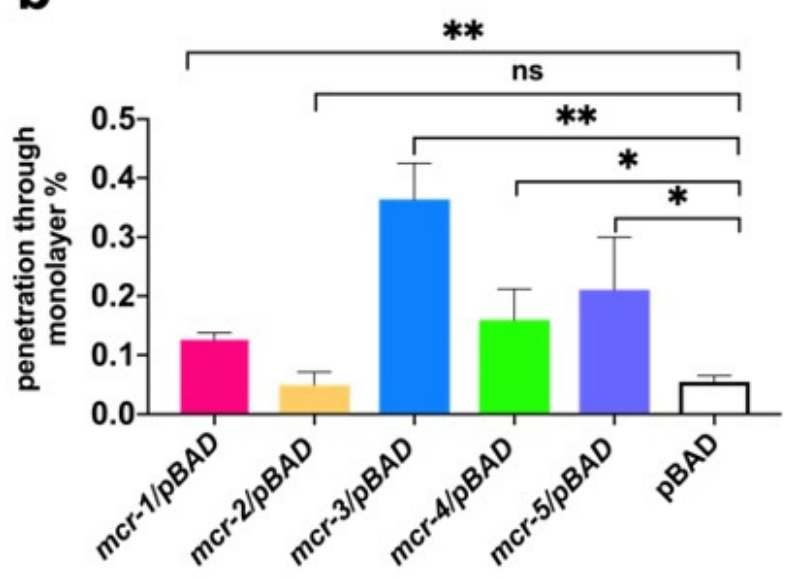

Figure 4. Bacterial virulence evaluated using trans-well assays and G. mellonella. (a) Study design of the trans-well assay. (b) Percentage of MCR-expressing strains that passed through the Caco- 2 cell monolayer at $2 \mathrm{~h}$ measured by viable counting. ${ }^{*} p<0.05,{ }^{* *} p<0.01$, ns: not significant.

We used G. mellonella to evaluate bacterial virulence in vivo. The movement abilities of $G$. mellonella infected with strains expressing MCR-1, $-3,-4$, and -5 were shown to be rapidly decreased, while the skin of the larvae quickly darkened within $1 \mathrm{~h}$ after injection (Figure 5a). In this model, MCR-2-expressing strains still showed similar results to those of the control group.

We detected the LPS concentrations in the fluid of ground G. mellonella. The G. mellonella infected with bacterial strains expressing MCR-1, $-3,-4$, and -5 contained significantly higher LPS concentrations in the larvae grinding fluid $(p<0.05)$ than did the MCR-2-expressing strains, which were similar to those of the control group injected with only pBAD-harboring strains (Figure $5 b$ ). 


\section{a}

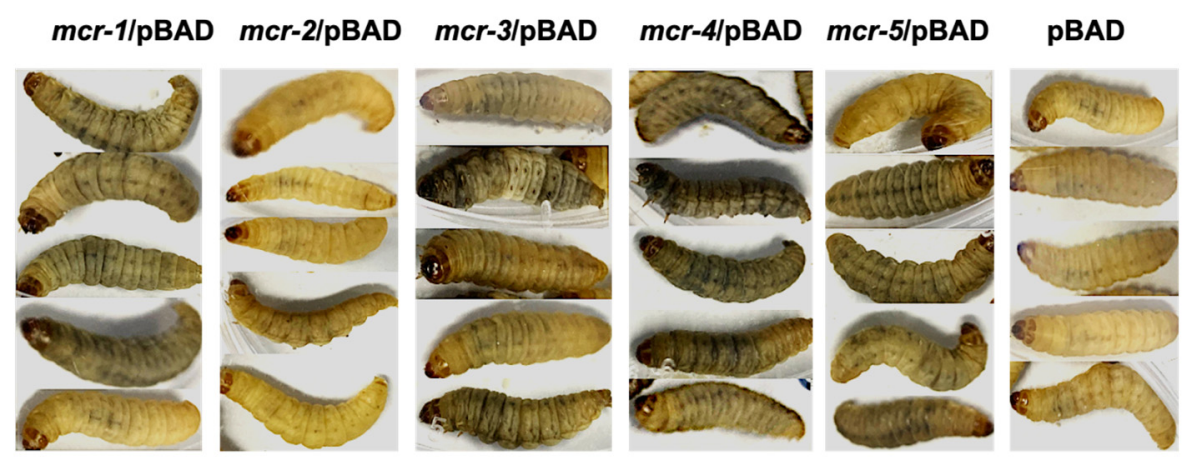

b

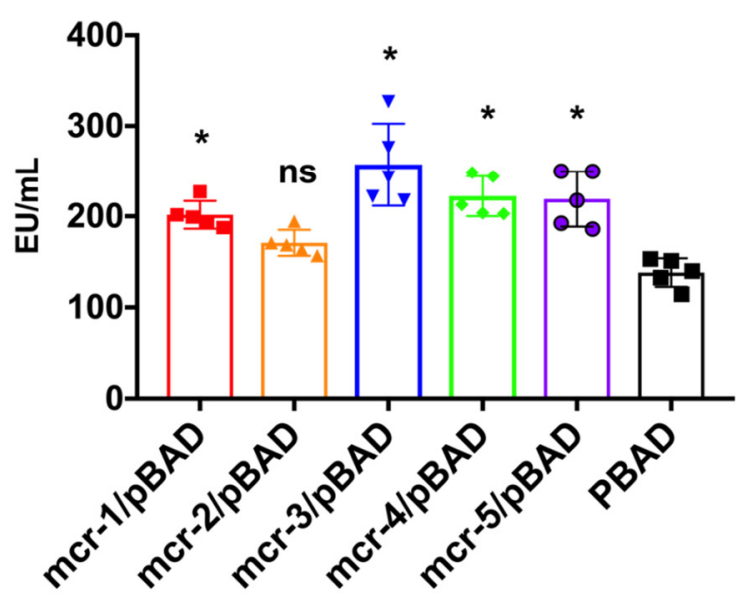

Figure 5. Bacterial virulence evaluated in a G. mellonella model. (a) Melanization of G. mellonella infected with MCR-expressing strains. (b) LPS concentrations in G. mellonella after infection. * $p<0.05$, ns: not significant.

\section{Discussion}

MCR family proteins catalyze an additional $\mathrm{pEtN}$ group on lipid $\mathrm{A}$, which results in attenuating the bacterium's affinity for colistin, and obviously compromises the efficacy of colistin as a last-resort antibiotic. Although $m c r$ genes $(m c r-1 \sim 5)$ belong to one family, their prevalence is significantly varied. Of $m c r-1 \sim 5, m c r-1$ is the most widely disseminated variant, however, there are only sporadic reports of $m c r-2 \sim 5$ which dissemination across a limited area [11]. Resistance-associated fitness costs may inhibit gene further spread in the absence of antibiotics. In our research, the expressions of MCR-3 5 conferred more severe fitness burdens on bacteria than those of MCR-1 and -2, suggesting that $m c r-1$ and -2 exhibit better mutual adaptation with the host bacteria (in our study is E. coli) than do mcr-3 5. On this basis, $m c r-1$ and -2 should have a similar prevalence level; however, $m c r-1$ is much more widespread than $m c r-2$. We further compared the growths of strains under colistin exposure; on this occasion, MCR-1-expressing strains showed the best growth among MCR$1 \sim 5$, which might explain the widespread of $m c r-1$ but not mor-2. Currently, most of the mcr-1-positive isolates are limited to Enterobacteriaceae. A global surveillance revealed that, among the 29 mcr-positive Enterobacteriaceae isolated from 2014 to 2016, 24 (82.75\%) carried $m c r-1$ or its variants [21]. A report on $m c r-1 \sim 5$ prevalence in Enterobacteriaceae showed that 54 of $61 \mathrm{mcr}$-positive isolates were E. coli [22]. Colistin-resistant E. coli isolates in food-producing animals in Italy during 2014-2015 were shown to carry $\mathrm{mcr}$ genes; among the $15 \mathrm{mcr}$-positive E. coli, 14 harbored $m c r-1$, and only one tested positive for $m c r-4$ [23]. The better mutual adaptation between $m c r-1$ and E. coli in terms of our result of varied 
fitness cost may partially explain why mor-1 is the most prevalent gene in the mor family. Inconsistent results about fitness cost conferred by MCR expression have been reported in K. pneumoniae and E. coli, with reduced biological fitness being observed [1,24]. However, $m c r-1$ was also reported as being able to confer fitness advantages to the host when carried by IncI 2 and IncX 4 plasmids, suggested different host strain and plasmid combinations may exert varied fitness effects. This might explain why IncI 2 and IncX 4 are the major vehicles of $m c r-1$ worldwide [15], as certain genes on these plasmids may help strains compensate for the fitness costs of MCR expression [25]. Except for fitness cost, the types of plasmids that carry mor genes could also result in large different in its distribution, as the globally distributed $m c r-1$ has been associated with the broad host range plasmids including IncI2, IncX4, and IncHI2 [13,26]; however, $m c r-2$ was currently only observed carried by IncX4 plasmid $[4,6]$. Whether there are other contributing factors for the widespread of $m c r-1$ will be explored in subsequent studies.

Acyl chains and phosphate groups of disaccharides are the major moieties that contribute to endotoxicity of lipid A [27]. Removing of phosphate groups from lipid A makes some bacterial species less toxic [28]. Studies have yielded conflicting results regarding how modifying lipid A with pEtN alters bacterial virulence. In this study, we used in vitro and in vivo models to understand the role of $\mathrm{mcr}$ genes in bacterial virulence. Strains overexpressing $m c r$ (except $m c r$-2) showed increased virulence compared with that of the control group. Our results were inconsistent with those of Yang et al., who reported that acquisition of naturally generated $m c r-1$ carried by wild-type plasmids in E. coli markedly depleted its virulence [1]. However, in their study, acquisition of the mcr-1-carrying plasmid did not affect bacterial growth, and the fitness cost was moderate. Thus, we inferred that the increased virulence observed in our research might be related to fitness costs generated by MCR overexpression. MCR-mediated modification of lipid A is reported to cause profound changes in the bacterial outer membrane architecture in E. coli [1]; subsequently, membrane modification may further result in important membrane component release and could increase bacterial virulence in vivo $[29,30]$. To test our hypothesis, we detected the LPS concentrations in G. mellonella after bacterial infection. G. mellonella infected with MCR-expressing strains showed higher tissue LPS concentrations than did the control group. The result supports our hypothesis that MCR overexpression mediated lipid A modification result in LPS release and increase bacterial virulence. Our results are similar with those of Kaito Chikara et al.'s report, where, by developing a silkworm infection model, the researchers found that non-pathogenic E. coli could acquire virulence by mutating essential gene in growth or LPS transport, and this mutation could increase the amount of LPS in the outer membrane vesicle fraction [31]. In our trans-well assay, we observed an increased amount of bacteria expressing MCR penetrate the in vitro epithelial barrier, as it was reported that lipid A remodeling could trigger LPS liberty and the liberated LPS could increase intestinal tight junction permeability [32,33]; thus, we hypothesized that MCR expressing may help bacteria destroy epithelial barrier function by remodeling bacteria membrane and liberating the LPS. Regarding virulence and fitness costs of MCR-expressing strains, we also noticed that strains with greater fitness burden tended to be more virulent; however, depending on the present study, we could not directly correlate the variation in bacterial virulence to the fitness costs. Hence, the mechanisms of the variations in $m c r$-mediated bacterial virulence are complex and remain unclear, and therefore further studies are needed.

Our study had some limitations. We observed phenotypic differences in fitness costs and virulence among mcr-1 5-carrying bacteria. However, we could not explain the reason for these differences. Hence, we hypothesized some potential reasons for these differences. First, the expressions of different MCRs are precisely regulated in host bacteria, and the transcriptional level of the plasmids that carry $\mathrm{mcr}$ is controlled by both the promoter and the genomic background of the host bacteria. This is because the $m c r-1$ carried by different plasmids and transformed in the same host strain (EC600) have shown the same expression 
at baseline [34]. Second, the previous reports confirmed different enzymatic efficiencies of different MCR proteins [35].

\section{Conclusions}

Collectively, we explored the effect of MCR-1 5 expression on host bacteria. Among MCR-1 5, MCR-1-expressing strains showed the best survival under colistin exposure and the moderate fitness costs, which might be a critical factor for the increased global dissemination of $m c r-1$. We also detected the varied effect of $\mathrm{mcr}$ genes on enhancing bacterial virulence, which may be a potential risk of increased infection along with compromising colistin efficacy. Thus, we should emphasize the importance of the $\mathrm{mcr}$ genes' threat to human and animal health, and more attention should be paid to deciphering prevalence and mechanism of $m c r$.

Author Contributions: J.S., Y.W., C.W., Z.L. (Zhihai Liu), W.Y. and W.L. participated in the experimental design. W.L., L.Y., L.Q., S.S., Z.L. (Zhuoren Ling) and R.Z. carried out experiments and wrote the manuscript. Z.L. (Zhihai Liu), W.Y., Y.W. and J.S. helped to revise the manuscript. All authors have read and agreed to the published version of the manuscript.

Funding: This work was supported in part by grants from the National Natural Science Foundation of China (81861138051 and 81991535).

Institutional Review Board Statement: Not applicable.

Informed Consent Statement: Not applicable.

Data Availability Statement: The data presented in this study are available on request from the corresponding author.

Conflicts of Interest: The authors declare no competing financial interest.

\section{Appendix A}

Table A1. Primers used in this research.

\begin{tabular}{lll}
\hline Primers & Sequences & Source \\
\hline$m c r-1-\mathrm{F}$ & ggctaacaggaggaattaaccatggATGATGCAGCATACTTCTGTGTG & This study \\
$m c r-1-\mathrm{R}$ & gccaaaacagccaagcttcgaattcTCAGCGGATGAATGCGGTG & This study \\
$m c r-2-\mathrm{F}$ & ggctaacaggaggaattaaccatggATGACATCACATCACTCTTG & This study \\
$m c r-2-\mathrm{R}$ & gccaaaacagccaagcttcgaattcTTACTGGATAAATGCCGC & This study \\
$m c r-3-\mathrm{F}$ & ggctaacaggaggaattaaccatggATGCCTTCCCTTATAAAAATAAAAATTG & This study \\
$m c r-3-\mathrm{R}$ & gccaaaacagccaagcttcgaattcTTATTGAACATTACGACATTGAC & This study \\
$m c r-4-\mathrm{F}$ & ggctaacaggaggaattaaccatggGTGATTTCTAGATTTAAGACGTTATC & This study \\
$m c r-4-\mathrm{R}$ & gccaaaacagccaagcttcgaattCTAATACCTGCAAGGTGC & This study \\
$m c r-5-\mathrm{F}$ & ggctaacaggaggaattaaccatggATGCGGTTGTCTGCATTTATC & This study \\
$m c r-5-\mathrm{R}$ & gccaaaacagccaagcttcgaattcTCATTGTGGTTGTCCTTTTC & This study \\
\hline
\end{tabular}

\section{References}

1. Yang, Q.; Li, M.; Spiller, O.B.; Andrey, D.O.; Hinchliffe, P.; Li, H.; MacLean, C.; Niumsup, P.; Powell, L.; Pritchard, M.; et al. Balancing mcr-1 expression and bacterial survival is a delicate equilibrium between essential cellular defence mechanisms. Nat. Commun. 2017, 8, 1-12. [CrossRef]

2. Wang, Y.; Tian, G.; Zhang, R.; Shen, Y.; Tyrrell, M.J.; Huang, X.; Zhou, H.; Lei, L.; Li, H.; Doi, Y.; et al. Prevalence, risk factors, outcomes, and molecular epidemiology of mcr-1-positive Enterobacteriaceae in patients and healthy adults from China: An epidemiological and clinical study. Lancet Infect. Dis. 2017, 17, 390-399. [CrossRef]

3. Voigt, A.M.; Ciorba, P.; Döhla, M.; Exner, M.; Felder, C.; Lenz-Plet, F.; Sib, E.; Skutlarek, D.; Schmithausen, R.M.; Faerber, H.A. The investigation of antibiotic residues, antibiotic resistance genes and antibiotic-resistant organisms in a drinking water reservoir system in Germany. Int. J. Hyg. Environ. Health 2020, 224, 113449. [CrossRef]

4. Wang, X.; Wang, Y.; Ying, Z.; Li, J.; Yin, W.; Wang, S.; Shen, J.; Shen, Z.; Wang, Y. Emergence of a novel mobile colistin resistance gene, mcr-8, in NDM-producing Klebsiella pneumoniae. Emerg. Microbes Infect. 2018, 7, 1-9. [CrossRef] [PubMed] 
5. Yin, W.; Li, H.; Shen, Y.; Liu, Z.; Wang, S.; Shen, Z.; Zhang, R.; Walsh, T.R.; Shen, J.; Wang, Y. Novel Plasmid-Mediated Colistin Resistance Gene mcr-3 in Escherichia coli. mBio 2017, 8, e00543-17. [CrossRef] [PubMed]

6. Carattoli, A.; Villa, L.; Feudi, C.; Curcio, L.; Orsini, S.; Luppi, A.; Pezzotti, G.; Magistrali, F.C. Novel plasmid-mediated colistin resistance mcr-4 gene in Salmonella and Escherichia coli, Italy 2013, Spain and Belgium, 2015 to 2016. Eurosurveillance 2017, 22, 30589. [CrossRef] [PubMed]

7. Borowiak, M.; Fischer, J.; Hammerl, J.A.; Hendriksen, R.S.; Szabo, I.; Malorny, B. Identification of a novel transposon-associated phosphoethanolamine transferase gene, mcr-5, conferring colistin resistance in d-tartrate fermenting Salmonella enterica subsp. enterica serovar Paratyphi B. J. Antimicrob. Chemother. 2017, 72, 3317-3324. [CrossRef] [PubMed]

8. AbuOun, M.; Stubberfield, J.E.; Duggett, A.N.; Kirchner, M.; Dormer, L.; Nunez-Garcia, J.; Randall, P.L.; Lemma, F.; Crook, W.D.; Teale, C.; et al. mcr-1 and mcr-2 (mcr-6.1) variant genes identified in Moraxella species isolated from pigs in Great Britain from 2014 to 2015. J. Antimicrob. Chemother. 2017, 72, 2745-2749. [CrossRef]

9. Xu, T.; Zhang, C.; Ji, Y.; Song, J.; Liu, Y.; Guo, Y.; Zhou, K. Identification of mcr-10 carried by self-transmissible plasmids and chromosome in Enterobacter roggenkampii strains isolated from hospital sewage water. Environ. Pollut. 2021, $268,115706$. [CrossRef]

10. Carroll, L.M.; Gaballa, A.; Guldimann, C.; Sullivan, G.; Henderson, L.O.; Wiedmann, M. Identification of Novel Mobilized Colistin Resistance Gene mcr-9 in a Multidrug-Resistant, Colistin-Susceptible Salmonella enterica Serotype Typhimurium Isolate. mBio 2019, 10, e00853-19. [CrossRef]

11. Ling, Z.; Yin, W.; Shen, Z.; Wang, Y.; Shen, J.; Walsh, T.R. Epidemiology of mobile colistin resistance genes mcr-1 to mcr-10. J. Antimicrob. Chemother. 2020, 75, 3087-3095. [CrossRef]

12. Yang, Q.E.; MacLean, C.; Papkou, A.; Pritchard, M.; Powell, L.; Thomas, D.; Andrey, O.D.; Li, M.; Spiller, B.; Yang, W.; et al. Compensatory mutations modulate the competitiveness and dynamics of plasmid-mediated colistin resistance in Escherichia coli clones. ISME J. 2020, 14, 861-865. [CrossRef]

13. Wu, R.; Yi, L.; Yu, L.; Wang, J.; Liu, Y.; Chen, X.; Lv, L.; Yang, J.; Liu, J. Fitness Advantage of mcr-1-Bearing IncI2 and IncX4 Plasmids in Vitro. Front. Microbiol. 2018, 9, 331. [CrossRef]

14. Da Silva, G.J.; Domingues, S. Interplay between Colistin Resistance, Virulence and Fitness in Acinetobacter baumannii. Antibiotics 2017, 6, 28. [CrossRef]

15. Bahador, A.; Farshadzadeh, Z.; Raoofian, R.; Mokhtaran, M.; Pourakbari, B.; Pourhajibagher, M.; Hashemi, B.F. Association of virulence gene expression with colistin-resistance in Acinetobacter baumannii: Analysis of genotype, antimicrobial susceptibility, and biofilm formation. Ann. Clin. Microbiol. Antimicrob. 2018, 17, 24. [CrossRef]

16. De La Tabla, V.O.; Ortega, A.; Buñuel, F.; Pérez-Vázquez, M.; Marcos, B.; Oteo, J. Detection of the high-risk clone ST131 of Escherichia coli carrying the colistin resistance gene mcr-1 and causing acute peritonitis. Int. J. Antimicrob. Agents 2017, 49, 115-116. [CrossRef]

17. Simon-Flament, S.; de Toro, M.; Mora, A.; García, V.; Meniño, G.I.; Díaz-Jiménez, D.; Herrera, A.; Blanco, J. Whole Genome Sequencing and Characteristics of mcr-1-Harboring Plasmids of Porcine Escherichia coli Isolates Belonging to the High-Risk Clone O25b:H4-ST131 Clade B. Front. Microbiol. 2020, 11, 387. [CrossRef] [PubMed]

18. Hayashi, W.; Tanaka, H.; Taniguchi, Y.; Iimura, M.; Soga, E.; Kubo, R.; Matsuo, N.; Kawamura, K.; Arakawa, Y.; Nagano, Y.; et al. Acquisition of mcr-1 and Cocarriage of Virulence Genes in Avian Pathogenic Escherichia coli Isolates from Municipal Wastewater Influents in Japan. Appl. Environ. Microbiol. 2019, 85, e01661-19. [CrossRef] [PubMed]

19. Liu, Y.; Wang, Y.; Walsh, R.T.; Yi, L.; Zhang, R.; Spencer, J.; Doi, Y.; Tian, G.; Dong, B.; Huang, X.; et al. Emergence of plasmidmediated colistin resistance mechanism MCR-1 in animals and human beings in China: A microbiological and molecular biological study. Lancet Infect. Dis. 2016, 16, 161-168. [CrossRef] [PubMed]

20. Li, J.; Shi, X.; Yin, W.; Wang, Y.; Shen, Z.; Ding, S.; Wang, S. A Multiplex SYBR Green Real-Time PCR Assay for the Detection of Three Colistin Resistance Genes from Cultured Bacteria, Feces, and Environment Samples. Front. Microbiol. 2017, 8, 2078. [CrossRef]

21. Wise, M.G.; Estabrook, M.A.; Sahm, D.F.; Stone, G.G.; Kazmierczak, K.M. Prevalence of mcr-type genes among colistin-resistant Enterobacteriaceae collected in 2014-2016 as part of the INFORM global surveillance program. PLoS ONE 2018, 13, e0195281. [CrossRef]

22. Gelbíčová, T.; Baráková, A.; Florianová, M.; Jamborová, I.; Zelendová, M.; Pospíšilová, L.; Koláčková, I.; Karpíšková, R. Dissemination and Comparison of Genetic Determinants of mcr-Mediated Colistin Resistance in Enterobacteriaceae via Retailed Raw Meat Products. Front. Microbiol. 2019, 10, 2824. [CrossRef]

23. Alba, P.; Leekitcharoenphon, P.; Franco, A.; Feltrin, F.; Ianzano, A.; Caprioli, A.; Stravino, F.; Hendriksen, S.R.; Bortolaia, V.; Battisti, A. Molecular Epidemiology of mcr-Encoded Colistin Resistance in Enterobacteriaceae From Food-Producing Animals in Italy Revealed Through the EU Harmonized Antimicrobial Resistance Monitoring. Front. Microbiol. 2018, 9, 1217. [CrossRef] [PubMed]

24. Wang, R.; Van Dorp, L.; Shaw, L.P.; Bradley, P.; Wang, Q.; Wang, X.; Jin, L.; Zhang, Q.; Liu, Y.; Rieux, A.; et al. The global distribution and spread of the mobilized colistin resistance gene mcr-1. Nat. Commun. 2018, 9, 1-9. [CrossRef]

25. Russell, N.D.; Corvalan, J.R.F.; Gallo, M.L.; Davis, C.G.; Pirofski, L.-A. Production of Protective Human Antipneumococcal Antibodies by Transgenic Mice with Human Immunoglobulin Loci. Infect. Immun. 2000, 68, 1820-1826. [CrossRef] 
26. Shen, Y.; Wu, Z.; Wang, Y.; Zhang, R.; Zhou, H.-W.; Wang, S.; Lei, L.; Li, M.; Cai, J.; Tyrrell, J.; et al. Heterogeneous and Flexible Transmission of mcr-1 in Hospital-Associated Escherichia coli. mBio 2018, 9, e00943-18. [CrossRef] [PubMed]

27. Di Lorenzo, F.; De Castro, C.; Silipo, A.; Molinaro, A. Lipopolysaccharide structures of Gram-negative populations in the gut microbiota and effects on host interactions. FEMS Microbiol. Rev. 2019, 43, 257-272. [CrossRef]

28. Wang, X.; Ribeiro, A.A.; Guan, Z.; Abraham, S.N.; Raetz, C.R.H. Attenuated virulence of a Francisella mutant lacking the lipid A 4'-phosphatase. Proc. Natl. Acad. Sci. USA 2007, 104, 4136-4141. [CrossRef] [PubMed]

29. Hurley, J.C. Antibiotic-Induced Release of Endotoxin: A Reappraisal. Clin. Infect. Dis. 1992, 15, 840-854. [CrossRef] [PubMed]

30. Hellman, J.; Roberts, J.D.; Tehan, M.M.; Allaire, J.E.; Warren, H.S. Bacterial Peptidoglycan-associated Lipoprotein Is Released into the Bloodstream in Gram-negative Sepsis and Causes Inflammation and Death in Mice. J. Biol. Chem. 2002, 277, 14274-14280. [CrossRef]

31. Kaito, C.; Yoshikai, H.; Wakamatsu, A.; Miyashita, A.; Matsumoto, Y.; Fujiyuki, T.; Kato, M.; Ogura, Y.; Hayashi, T.; Isogai, T.; et al. Non-pathogenic Escherichia coli acquires virulence by mutating a growth-essential LPS transporter. PLoS Pathog. 2020, 16, e1008469. [CrossRef] [PubMed]

32. Guo, S.; Al-Sadi, R.; Said, H.M.; Ma, T.Y. Lipopolysaccharide Causes an Increase in Intestinal Tight Junction Permeability in Vitro and in Vivo by Inducing Enterocyte Membrane Expression and Localization of TLR-4 and CD14. Am. J. Pathol. 2013, 182, 375-387. [CrossRef]

33. Elhenawy, W.; Bording-Jorgensen, M.; Valguarnera, E.; Haurat, M.F.; Wine, E.; Feldman, M.F. LPS Remodeling Triggers Formation of Outer Membrane Vesicles in Salmonella. mBio 2016, 7, e00940-16. [CrossRef] [PubMed]

34. Zhang, H.; Miao, M.; Yan, J.; Wang, M.; Tang, Y.-W.; Kreiswirth, B.N.; Zhang, X.; Chen, L.; Du, H. Expression characteristics of the plasmid-borne mcr-1 colistin resistance gene. Oncotarget 2017, 8, 107596-107602. [CrossRef] [PubMed]

35. Li, H.; Yang, L.; Liu, Z.; Yin, W.; Liu, D.; Shen, Y.; Walsh, R.T.; Shao, B.; Wang, Y. Molecular Insights into Functional Differences between mcr-3- and mcr-1-Mediated Colistin Resistance. Antimicrob. Agents Chemother. 2018, 62. [CrossRef] 